\title{
THE MANAGEMENT OF INFANTS \\ WITH TRACHEO-OESOPHAGEAL FISTULA
}

Gorwon M. Wyant, F.r.C.P.(c), F.F.A.R.C.S., ANd Robert W. Cram, F.R.C.s.(c) *

OF ALL the congenital anomalies found in the newborn, none presents a greater challenge to the surgical team than the correction of tracheo-oesophageal fistula. Because of a number of problems peculiar to this condition which influence the anaesthetic and postoperative management and which are superimposed upon the problems of anaesthesia in the neonate in general, a detailed description of the management of these cases does not seem unwarranted.

\section{EMBRYology AND ANATOMY}

The various anomalies, clasşed under the common term of "tracheo-oesophageal fistula," arise between the third and sixth week of embryonic life. While the exact mechanism is not clearly understood, it is believed that the fistula is the outcome of an abnormality in the development of the laryngotracheal groove. This is an invagination on either side of the single tube from which both oesophagus and trachea are subsequently derived. The oesophageal atresia on the other hand is believed by some to be the result of incomplete canalization of the originally solid tube which is the precursor of the oesophagus. Others believe that vascular abnormalities in this region, associated with the primitive large vessels of the thorax, may be a factor in the causation of the atresia. Since atresia and fistula thus are the result of different developmental aberrations, it follows that tracheooesophageal fistula can occur without oesophageal atresia and vice versa.

A number of anatomic variations of tracheo-oesophageal fistula have been described, but in over 90 per cent of cases one finds an upper blind pouch, with a fistula connecting the lower portion of the oesophagus to the trachea in the region of the carina (Fig. 1A). In approximately 3 per cent of cases atresia of the oesophagus occurs without fistula (Fig. 1B). In a third variety, which comprises 2 per cent, a fistula (or fistulae) is present, the oesophagus being in continuity and patent (Fig. 1C). One per cent of cases have a fistulous communication between the upper pouch and the trachea, with a blind lower end (Fig. 1D). The least comnon variety is one in which both upper and lower pouch communicate with the trachea by means of a fistulous track (Fig. 1E). ${ }^{1}$ The following discussion of management is based primarily on the common variety. The most difficult abnormality to detect clinically is the one in which continuity of the oesophagus is maintained.

Close to 30 per cent of all patients with tracheo-oesophageal fistula have other major associated anomalies. ${ }^{2}$ The most common of these are of a cardiovascular or gastro-intestinal nature such as coarctation of the aorta, vascular ring, patent

"From the Departments of Anaesthesia and Surgery, University of Saskatchewan, and University Hospital, Saskatoon.

Can. Anaes. Soc. J., vol. 10, no. 2, March, 1963 

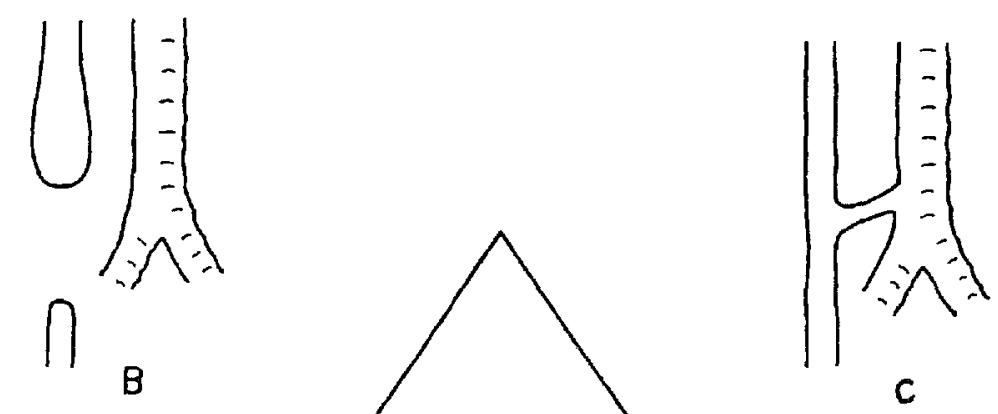

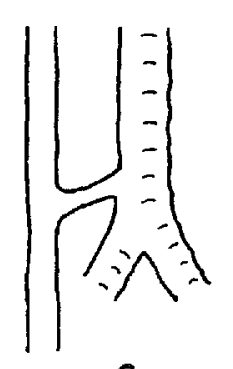

c
D

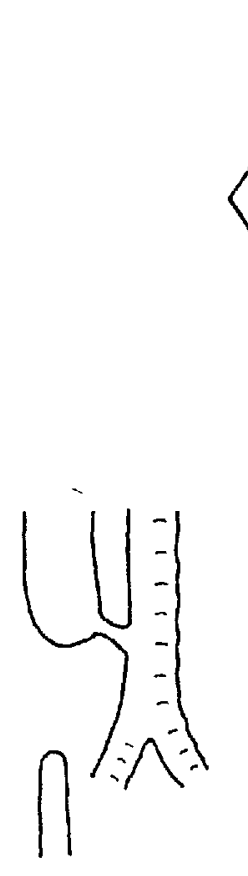

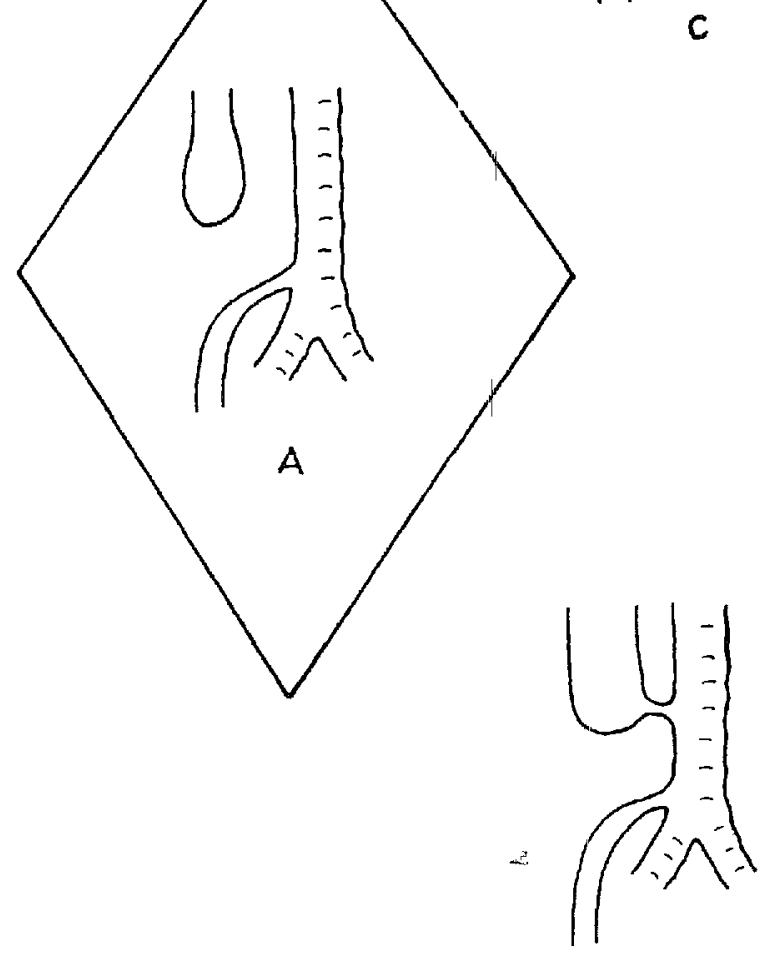

E

Frgure 1. Tyes of tracheo-oesophageal fistula. A. Common type: blind upper pouch; fistula between lower end and trachea, close to carina. B. Atresia of oesophagus without fistula (upper pouch usually reaches further down than in type $A$ and lower end may reach barely beyond diaphragm). C. Fistula with oesophagus in continuity. D. Fistula between upper pouch and trachea. E. Double fistula, one from each blind oesophageal and to trachea.

ductus arteriosus, imperforate anus, congenital hypertrophic pyloric stenosis, and duodenal atresia. Less commonly, mongolism, hydrocephalus, craniostosis, polycystic kidney, and others may be encountered.

\section{DiAgnosis)}

Much of the success or failure of the subsequent surgical repair rests with early diagnosis. The earlier the condition is recognized after birth the greater is the likelihood that serious contamination of the lungs from spill-over of saliva or milk and from regurgitation of gastric secretions via the fistula has not yet taken place and that atelectasis and aspiration pneumonitis have not yet advanced to the point where they will endanger seriously the success of the operation. Since 
tracheo-oesophageal fistula is frequently associated with hydramnios and prematurity, the obstetrician should forthwith exclude the presence of oesophageal atresia in all cases of hydramnios. Furthermore excessive salivation, and later the triad of bouts of choking, coughing, and cyanosis at the time of feeding, should suggest the diagnosis. If oesophageal atresia is present, a No. $10 \mathrm{or}^{-12} \mathrm{~F}$ catheter, passed into the oesophagus, will confirm the diagnosis in that the tube will be held up in the upper pouch some $10 \mathrm{~cm}$. from the upper gum. This can further be confirmed by plain X-rays, which frequently will demonstrate the blind upper pouch (Fig. 2). The diagnosis can be confirmed further by the careful instillation

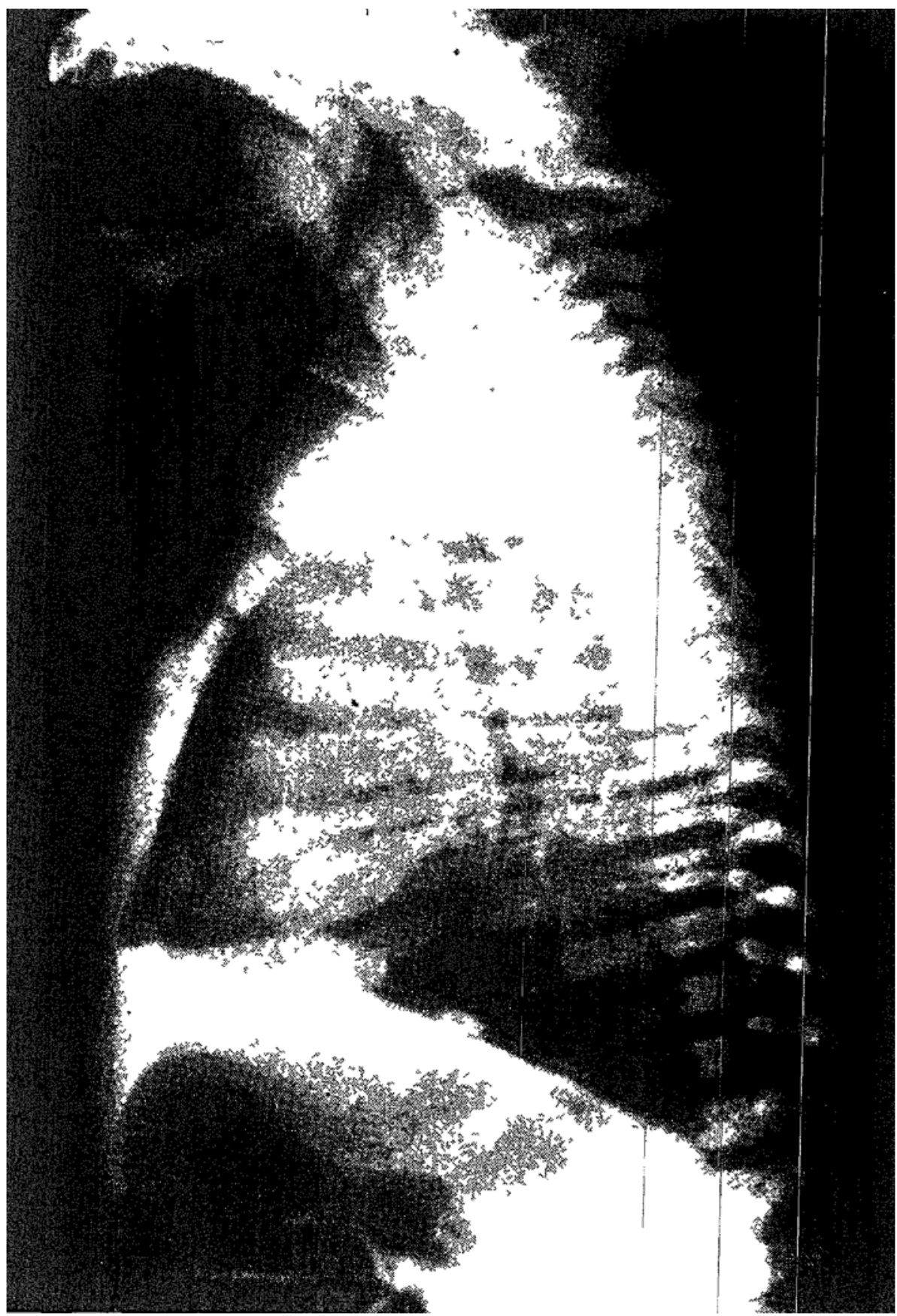

Figure 2. Plain X-ray showing upper pouch (note also air in G.I. tract). 
of a small quantity of aqueous contrast medium into the upper pouch ( $F_{1 g} 3$ ) this must be removed by suction as soon as it has senved its purpose and before it has spilled over into the tracheo bronchial tree These $\mathrm{A}$ rays should mclude both thora and abdomen since presence of arr in the stomach is usually asso ciatcd with the presence of a lower fistula unless thus is unusually narrow

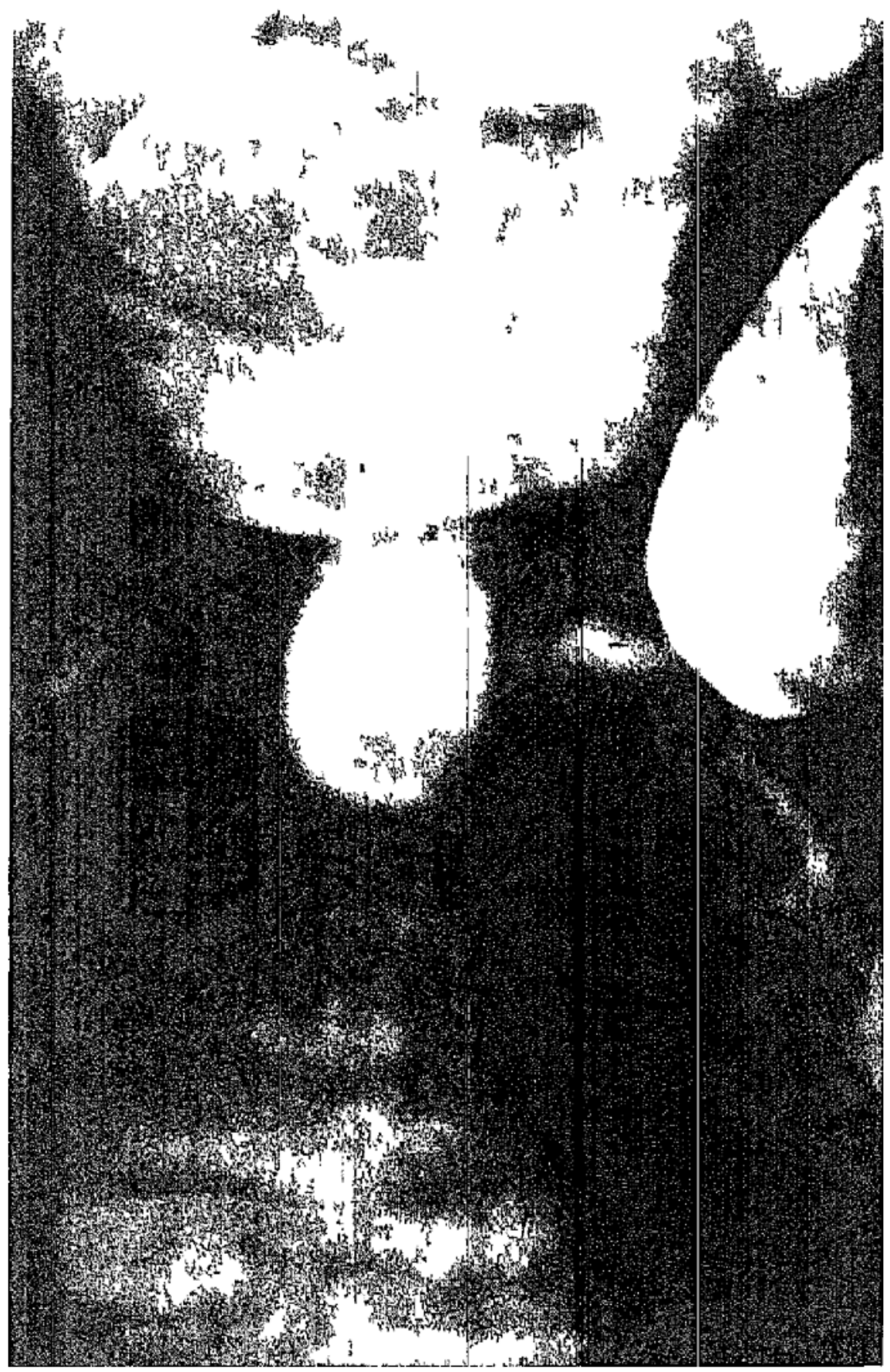

Figtre 3 Upper pouch demonstrited by mens of contrat medium 
Presence of air throughout the intestinal tract will rule out other intestinal atresias. If a suspicion of an intestinal atresia exists, both the tracheo-oesophageal fistula and the abdominal condition must be dealt with in the same operation, a situation which will markedly increase the risk to the infant. In summary, early diagnosis without previous feeding, maturity, and absence of other congenital anomalies improve the prognosis while the opposite of any or all of these worsens the outlook considerably.

\section{Anaesthesia and Operative Management}

As soon as the infant is first seen, it is placed into a head-up position to minimize aspiration. Half an hour before operation, atropine $0.2 \mathrm{mg}$. is administered intramuscularly and a cutdown is performed in one leg at the medial malleolus. The largest-diameter polyethylene catheter that will pass should be used and great care must be taken that it will continue to function satisfactorily throughout the operation. A breákdown of this cutdown and the consequent inability to replace blood-loss meticulously may have the most serious consequences. It must be realized that the newborn seven-pound infant has a circulating blood volume of approximately $280 \mathrm{ml}$. $(40 \mathrm{ml}$./ $/ \mathrm{lb}$.) and the premature infant has correspondingly less. Thus the unreplaced loss of 10 or $20 \mathrm{ml}$. of blood constitutes a considerable proportion of the infant's circulating volume.

On arrival in the operating room the infant is placed on a hyper-hypothermia blanket so that by this means the patient's body temperature can be maintained as near normal as possible. After insertion of the laryngoscope, the hypopharynx is emptied of excess mucus by gentle suction with a polyethylene catheter. Then endotracheal intubation is carried out with the infant awake. The head-up position is maintained until the tube is in place. Great care must be taken not to force too large an endotracheal tube past the narrow cricoid ring. In the fullterm infant we prefer a No. $15 \mathrm{~F}$ armoured tube whereas in smaller infants a Cole endotracheal tube is preferred. Immediately after intubation, the anaesthetist auscultates both lungs to ascertain that the endotracheal tube has not entered one of the main stem bronchi. The tube is then secured with tape and anaesthesia is induced with any agent with which the anaesthetist is most familiar. We have preferred halothane and oxygen for both induction and maintenance in our more recent cases, administering the agent by means, of either a Fluotec Mark II or a Vernitrol vaporizer. Depending upon the anaesthetist's prefefence and experience, cyclopropane or diethyl ether are equally satisfactory, although with the latter induction is more prolonged. As for the system of "administration, individual preference and familiarity with the equipment are the deciding factors. An Infant Circle Absorber, To-and-Fro absorption, the modified Ayre T-piece with breathing bag, or the Ayre T-piece alone may be used. Personally, we prefer the To-and-Fro method with large gas flow and open bag-tail. Presence of a bag facilitates ventilation of the lungs and affords better appreciation of changes in the patency of the airway caused by secretions and changes in lung compliance. However, adequate ventilation can also be achieved by simple intermittent occlusion of the open end of an unmodified Ayre T-piece. The anaesthetist now applies an infant blood-pressure cuff, inserts a thermocouple into the rectum for 
temperature monitoring, and attaches an electrocatdioscope by means of needle electrodes. The latter is preferred by us to the precordial stethoscope since it is more accurate, an oesophageal stethoscope not being indicated for obvious reasons. The infant is now turned into the left lateral position preparatory to performing a right lateral thoracotomy. Although the majority of paediatric surgeons now prefer the trans-pleural approach, the technique of anaesthesia is identical for the extra-pleural approach, especially in view of the fact that in a high percentage of extra-pleural operations the pleural cavity is inadvertently entered anyway sooner or later. We consider that frequent measurements of blood pressure, continuous observation of pulse-rate and rhythm by means of the cardioscope, monitoring and regulation of body temperature, and meticulous blood and fluid replacement are of the utmost importance in these patients. In a modern air-conditioned operating room relatively profound hypothermia is likely to develop in the course of a long operation and should be prevented. On the other hand, if the closed rebreathing method of anaesthesia is used and the operating room is not air-conditioned, dangerous hyperthermia may develop. Because of the narrow diameter of the veins in these tiny patients, blood is best administered by syringe, a three-way stopcock having been inserted previously into the infusion system close to the head of the table. Hence, we feel that two anaesthetists are essential for the conduct of this procedure. It is impossible for one individual to carry out ventilation, administer blood by syringe, regulate the blanket temperature, take blood-pressure readings, and accurately record these findings.

Only when all monitoring devices have been applied and are working satisfactorily are drapes applied in such a way that the anaesthetist retains access to the baby's head. A flashlight at the head of the operating table will enable him to observe the baby's colour. Anaesthesia is kept as light as possible and respiration is gradually controlled so that complete control has been established before the pleura is entered. This is not difficult to achieve in these small infants and, as a rule, does not require the use of muscle relaxants. The level of anaesthesia should be sufficiently light that the baby never loses all tone in the muscles of the hand and retains a slight degree of resistance to the forcible opening of the clenched fist.

The surgeon now begins the thoracotomy and takes great care to minimize blood-loss. An accurate count is kept of this by means of weighing sponges and the anaesthetist attempts never to fall behind significantly in blood replacement. While from now on the operation and the anaesthesia may progress uneventfully, serious and life-threatening complications may arise at any time with great suddenness and therefore constant vigilance is absolutely essential. Most of the diffculties encountered are peculiar to this condition and derive from the fact that some portion of the lungs may have been atelectatic from previous aspiration, that considerable amounts of gases may escape via the fistula into the gastrointestinal tract, that an abnormal amount of secretions is usually present in the tracheobronchial tree, and that there is difficulty in removing them through a very small endotracheal tube. Also small amounts of blood may enter the tracheobronchial tree at the time the fistula is being closed and kinking of the bronchial tree may occur at this stage, while partial collapse of the right lung must be 
maintained in order to provide room for the surgeon to complete the delicate task of anastomosing the oesophagus. Adequate suction must be available at all times as well as spare endotracheal tubes. If ventilation cannot be maintained at any time because of inspissated secretions or blood, no time must be lost in replacing the endotracheal tube if it is felt that it has become obstructed. This is difficult in an infant in the lateral position under drapes but it must be accomplished promptly as a lifesaving procedure. With closure of the fistula, the loss of gases into the gastro-intestinal tract ceases and, if that has been substantial, the anaesthetic must be adjusted to this new situation. All through the operation the blood pressure is monitored assiduously since these young infants do not tolerate prolonged hypotension. As the upper pouch is being mobilized, the anaesthetist will attempt to pass a fine catheter into the pouch; this is advanced later by the surgeon into the stomach, and over it the anastomosis is fashioned. Here again, the anaesthetist has need for $\mathbf{a}_{5}$ second pair of hands since it is impossible for one person to pass this tube and at the same time maintain adequate ventilation of the patient's lungs and perform all the other tasks required of him. Immediately upon conclusion of the operation, the infant should be fully awake, breathing spontaneously and adequately. After final tracheobronchial aspiration, the trachea is extubated and the patient is transferred to a pre-warmed high-humidity Isolette.

\section{Postoperative Management}

With the successful conclusion of the operative procedure itself, one now enters the critical first forty-eight postoperative hours. Again most difficulties during this period are associated with respiratory complications due to the immature state of the respiratory mechanism in the newborn, complicated by thoracotomy, contamination of the lungs, and secretions in the tracheobronchial tree. The infant is nursed in head-up position to obviate undue regurgitation and to facilitate adequate tidal exchange, and is kept in an atmosphere of supersaturated humidity to the extent where a continuous mist is maintained on the inner surface of the incubator, so that the baby is scarcely visible. Frequent turning from side to side is mandatory. Skilled nursing staff, familiar with the problems of these cases, is essential and supervision must be absolutely continuous twenty-four hours a day. While the surgeon and the anaesthetist will spend many hours with or in the immediate vicinity of the infant, they are usually unable to be present all the time; therefore, nurses must be alert to the imminence and $/$ severity of any abnormal manifestations. Gentle pharyngeal suction with a soft rubber catheter must be carried out frequently by the nursing staff. If necessary, because of respiratory embarrassment, direct suction of the tracheobronchial tree is carried out aseptically by the anaesthetist. However, this cannot be done too often since the repeated irritation of the respiratory mucosa may result in subglottic oedema and thus further embarrassment to respiration. If bouts of cyanosis occur and are repeated, tracheostomy is indicated and should be carried out without undue delay. While it is appreciated that this operation carries its own risks of complications, it has proved lifesaving on some occasions. Great care must be taken to prevent the tracheostomy tube from entering one of the main stem bronchi (most 
tracheostomy tubes for infants are too long) and it is well to have the standard tubes shortened for use in these very small infants. It is preferable that the diameter of the tracheostomy tube be not too wide so that the infant will still be able to breathe around it, as evidenced by the fact that some phonation is still present. If this can be maintained, the infant will not lose the co-ordinated use of his laryngeal muscles and removal of the tracheostomy tube after a few days is possible. If the tube is maintained longer and the infant is no longer accustomed to the use of his larynx, it may be necessary to retain the tube for weeks and even months since its removal may otherwise be accompanied by acute asphyxia. Meanwhile all necessary steps must be faken to control aspiration pneumonitis by the usual measures.

Another frequent cause of sudden postoperative catastrophe is the development of pneumothorax or even tension pneumothorax. This may occur on the side of thoracotomy as a result of leakage from the site of the previous fistula in the presence of a blocked underwater seal drainage; it requires immediate correction. Likewise development of pneumothorax on the opposite side is not unknown and it is then usually due to the rupture of alveoli from unduly vigorous ventilation by the anaesthetist. This is most frequently' seen when complications have arisen in the course of the operation and desperate efforts have been made to restore unimpeded ventilation. Again this condition must be dealt with immediately by the insertion of an underwater seal. Since these complications can arise suddenly at any time during day or night, it is of the utmost importance that they be recognized immediately and that all members of the surgical team remain available at any hour to deal with these contingencies without any delay whatsoever.

For the first few days the infant is maintained on intravenous fluids but after two days the gastric tube may be withdrawn and again two or three days latèr, after a diodrast swallow has established patency of the anastomosis, small and repeated oral feeds may be started, as recommended by the paediatrician. Once the critical first forty-eight hours have passed, no major life-threatening complications are likely to occur except for those created by anastomatic leaks. This is a possibility up to the tenth postoperative day and therefore the close supervision of the infant in the intensive therapy area is maintained for at least that length of time.

\section{SUMMARY}

Our technique of dealing with the problems associated with repair of tracheooesophageal fistula has been described as we have developed it over the years. This method employs basic principles of paediatric anaesthesia and takes cognizance of the many additional factors which present themselves in this condition. These are due primarily to involvement of the respiratory tree in the disease, and the frequent coexistence of prematurity and other life-threatening congenital anomalies. With an incidence variously reported as 1:1,300 to 1:4,500 of live births, it is obvious that the condition is relatively infrequently encountered in the average general hospital. In an operation beset with so many problems and pitfalls, in which meticulous attention to even the most minor detail is essential 
and which is seen at most six times a year in any but the largest general hospitals or in children's hospitals, it is essential that for best results the management should be limited to one team, so that avoidable mistakes are not repeated by combinations of different individuals. When one thinks in terms of a team, this must, in addition to surgeon and anaesthetist, include the nurses in the intensive treatment area upon whose skill and acute appraisal of abnormal situations the survival of these infants depends to a large measure in the postoperative period. The paediatricians contribute by their advice on fluid replacement therapy and other non-surgical problems and they regulate the oral feeding regimen until such time as the infants are returned to their primary care during convalescence. Until then, all treatment orders are written by one person only (usually by the surgeon or by his resident under his direct orders), after due consultation with other members of the team. This avoids the confusion arising out of contradictory orders or the omission of others, and ensures that at least one person is completely in the picture at all times of every phase of treatment.'

\section{RÉSUMÉ}

Notre conduite en présence d'une fistule trachéo-oesophagienne se base sur une collaboration étroite entre le chirurgien, l'anesthésiste, le pédiatre et l'équipe d'infirmières. Les principaux problèmes dans ces circonstances sont: la prématurité fréquente, les complications pulmonaires aussi bien avant qu'après l'opération et la grande fréquence d'anomalies congénitales additionnelles qui peuvent menacer la vie de l'enfant.

Au cours de l'opération, nous enregistrons les signes cardiovasculaires, la $\mathrm{T}^{0}$, les pertes de sang, et nous prenons les mesures nécessaires pour éviter tout écart de la normale. Pour atteindre ce but, nous plaçons l'enfant dans une couverture hyper- ò hypo-thermisante et nous installons une électrode de thermocouple dans le rectum et des électrodes de cardioscope. Avant l'opération, nous faisons une dissection veineuse afin de remplacer bien adéquatement le sang perdu. Nous pratiquons l'intubation trachéale - l'enfant étant éveilleé - et aspirons les sécrétions présentes dans l'hypopharynx. Il faut faire attention pour éviter l'intubation bronchique involontaire. N'importe quel agent anesthésique reconnu et n'importe quelle méthode d'administration peuvent être utilisés, à la condition qu'on respecte les principes de base de l'anesthésie pédiatrique. Durant toute l'opération, nous recommandons la plus étroite surveillance, car il peut survenir des complications très soudainement, principalement sur le système respiratoire, et si elles ne sont pas diagnostiquées et traitées aussitôt, elles peuvent menacer la vie de l'enfant. C'est pour cette raison et à cause des nombreux détails nécessaires pour maintenir l'enfant dans une condition optima que l'auteur recommande que, pour de tels cas, deux anesthésistes soient disponibles. Au cours des suites opératoires il peut survenir de nombreuses et sérieuses complications, d'où la nécessité d'entourer l'enfant de la plus étroite surveillance à tous les instants par une équipe d'infirmières habiles et, également, par le chirurgien et/ou l'anesthésiste. Les deux plus fréquentes complications observées au cours des suites opératoires immédiates sont: une obstruction respiratoire et un pneumothorax; toutes les deux requièrent des soins immédiats. De temps en temps, il peut être 
indiqué de pratiquer une trachéotomie pour assurer une ventilation adéquate, pendant que l'on traite le pneumothorax de la façon ordinaire. Plus tard, c'est la fistule au niveau de l'anastomose qui constitue le plus grand risque.

\section{ACKNOWLEDGMENT}

The authors acknowledge with thanks the assistance of Dr. Manuel Ty, F.R.C.s.(c), who made the line-drawings of Figure 1.

\section{REFERENCES}

1. Swenson, O. Pediatric Surgery, 1st ed. New York: Appleton-Century-Crofts, Inc. (1958).

2. Benson, C. D. et al. Pediatric Surgery, Vol. 1, Ist ed. Chicago: Year Book Medical Publishers, Inc. (1962). 